
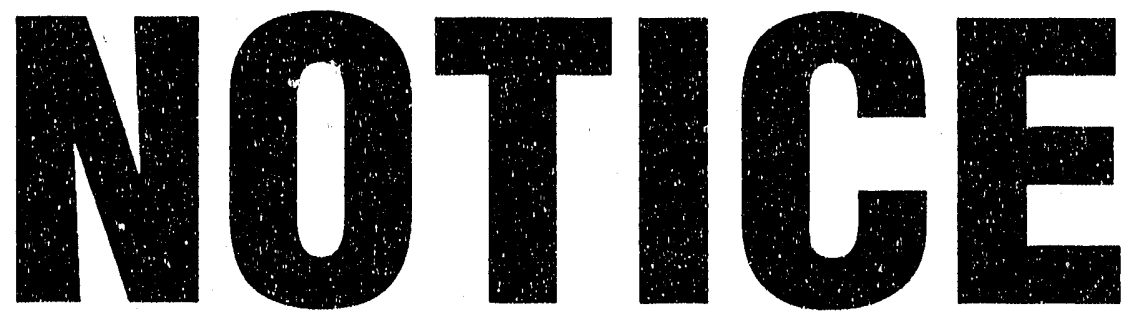

CERTAIN DATA
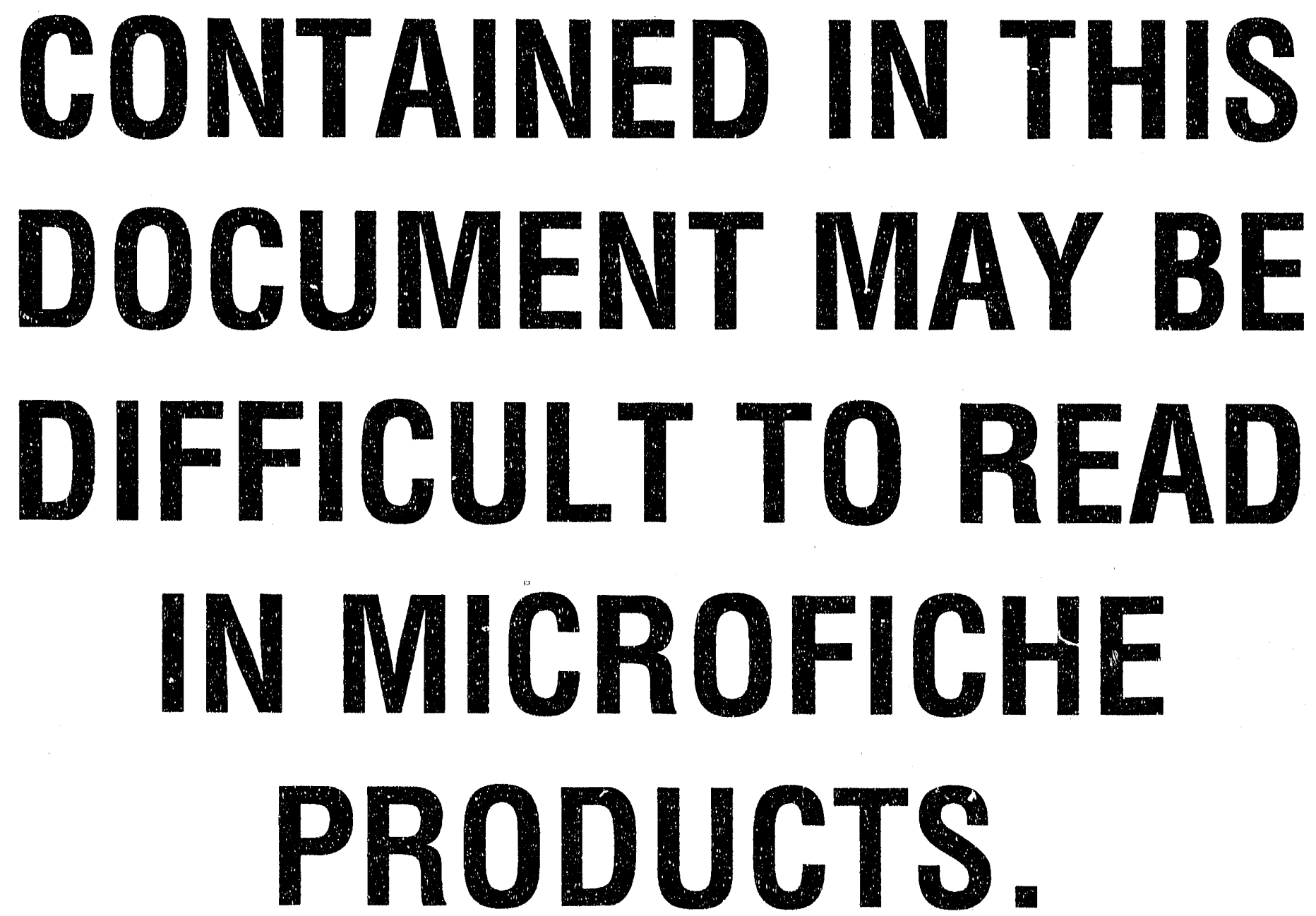


\title{
DISCLAIMER
}

This report was prepared as an account of work sponsored by an agency of the United States Government. Neither the United States Government nor any agency thereof, nor any of their employees, makes any warranty, express or implied, or assumes any legal liability or responsibility for the accuracy, completeness, or usefulness of any information, apparatus, product, or process disclosed, or represents that its use would not infringe privately owned rights. Reference herein to any specific commercial pruduct, process, or service by trade name, trademark, manufacturer, or otherwise does not necessarily constitute or imply its endorsement, recommendation, or favoring by the United States Government or any agency thereof. The views and opinions of authors expressed herein do not necessarily state or reflect tilose of the United States Government or any agency thereof.

\section{A Recoil Mass Spectrometer for the HHIRF Facility}

J. D. Cole

Idaho National Engineering liaboratory

E. G. \& G.

Idaho Falls, ID 8341.5

T. M. Cormier

Department of Physics and Cyclotron Institute

Texas A \& M University

College Station, TX 77843

J. H. Hamilton

Department of Physics and Astronomy

Vanderbilt University

Nashville, TN 37230
EGG-M- -89379

DE91 001840

NOV 051990

\section{ABSTRACT}

\begin{abstract}
A Recoil Mass Spectrometer (RMS) is to to thilt that will carry out a hroad rescarch program in heavy-ion science. The RMS will make possitle the study of entherwise inacesessible exotic nuclei. Careful attention has been given to match the RMS to all the heams available from the HHIRF accelerators, including those heams with the highest energy, as well as massive particles for use in inverse reactions. The RMS is to he a momentum achromat followed by a split electric-dipole mass spectrometer of the type operating at NSRL at the University of Rochester. The RMS is essential for many of the proposed experiments on short-lived and/or low cross-section products. The spectrometer design is discussed, with examples and comparisons with other spectrometers given. Detector arrays to he used with the RMS are also discussed.
\end{abstract}

\subsection{INTRODUCTION}

For many years scientists have studied nuclei far off stability, both via their radioactive decays and in-beam $y$-ray spectroscopy. (Several reviews ${ }^{1-3}$ have examples of work relevant to this discussion.) As the regions of known nuclei are pushed out (o) more neutron deficient nuclei, the cross-sections for their production in heavy-ion reactions are so small $(\leqslant$ a few mb) that they are difficult or impossible to study by traditional in-beam $y$ ray techniques. However, there are important physics questions to be answered by studies of these lighter nuclei. New ways have been sought 10 identify them.

Several groups have responded by recoginzing the power of a Recoil Mass Spec. trometer (RMS). The Daresbury RMS4 is operational (yielding exciting results); the RMS at Legnaro 5 (LNL), Italy, and the RPMSt at MSU are becoming operational; and others. such as Argonne National Laboratory's Fragment Mass Analyzer7 (FMA) and Texais Adix University's MARS project, 8 are being built. 
where the first successful RMS of the current design was develoned," studies of nuclei far from stability that have production cross-sections $x 1 \mathrm{mb}$ have been underwaly for several years. This spectrometer was combined with a segmented neutron detector ${ }^{10}$ and Gic detectors to produce a powerful tool for in-beam spectroscopy. These in-beam y-ray recoil-mass coincidence studies were the first ones done. As noted by the Datreshury gromp when these results were first reported, 1 the results clearly justified their large effort. Included in the initial studies were the identification for the first time of levels ${ }^{2}$ in $73 \mathrm{Br}$ and the extension to higher spins of the known hands in a number of nucleit1, 12 in this region. The success of these studies strongly encouraged the expansion of the research with the Rochester RMS.

Nevertheless, the Rochester RMS had limitations, including the energy of the accelerator, which limited the range of the nuclei far off stability that could be reached. This has been improved with the upgraded energy of the Rochester tandem, but there are still regions of heavy nuclei far from stability that will not he accessible hecause of heam energy. More importantly, the rigidity of the Rochester RMS was not designed to cover inverse reactions, where one uses a heavy projectile on a light target. The inverse reactions are important when very-low cross-section products are to be studied, hecause kinematic focusing can increase their intensity through the RMS hy large factors and because the high-velocity recoils allow $Z$ identification in a $\Delta E$ detector, hoth of which can make the difference between success and failure in an experiment. Thus, the idea for an RMS, which would be connected to the higher-energy accelerators at the Holifield Heavy Ion Rescarch Facility and which would be capable of separating products in inverse reactions, was born.

The key design features which were established for the RMS to be operated at HHIRF were the following: 1) To match as well as possible the RMS rigidity to the beams and energies available from the HHIRF accelerators, especially including those for inverse reactions. 2) To make the solid angle as large as possible to study very-weak reaction channels. 3) To make the spectrometer flexible to cover broad ranges of different research areas, both for now and for the future. The RMS achieves these goals, and it will be an important facility in the world for research with such devices.

\subsection{SPECTROMETER DEVELOPMENT}

The recoil mass spectrometer described here is designed to analyze heavy nucleat products from a heavy-ion-induced reaction. Several papers ${ }^{13-15}$ give reviews of spectrom. eters used in nuclear physics, including recoil mass spectrometers. The intent here is not to reproduce those discussions but to point io the important factors that make an RMS, and in particular the RMS being constructed for HHIRF, unique in its abilities. Comparisons: with specific spectrometers are made to emphasize particular points.

Various spectrometers (principally magnetic in character) have been used since the discovery that radioactive decay involved the emission of particles that could be manipulated by fields. Although the need for high-quality spectrometers dates from the early development of accelerators, it was not until the late 1940 's that modern spectrometers began to be designed. There has always been particular interest in determining the $Z$ or element number of the reaction products. The $Z$ cannot be determined by electric or magnetic deflection alone; however, in an inverse reaction, detectors capable of determining $Z$ within one unit can be used at the focal plane of the spectrometer. Omitting $Z$ from further discussion, the properties that can be determind are the mass mumer $(A)$, the kinctic energy $(E)$ and in come nacas tho mom.. .1 . $T$. 
the recoil $(q=Q e)$, its velocity $(v)$, and its mass $(m)$ are the parameters that determine its path through a spectrometer. Magnetic devices disperse in momentum $(p / q)$, and electric ones disperse in energy $(E / q)$. A combination of these two element. leads to a focusing of lines of constant $m / q$ on the focal plane of a spectrometer. This is, in essence, the approacin taken with the development of the current family of recoil mass spectrometers.

The direction of the development has been influenced by several problems that must be solved to provide good identification of the nuclear products. The direct identification of the products has been limited by two prohlems. First, the reaction preducts and the elastically-scattered beam particles usually both lie in the forward direction. For a simple particle detector, this gives a high gross count rate that masks the low count rate of the reaction products. The second problem for detectors alone is that, for the more-massive recoils $(A \approx 100)$, the energy resolution of the detectors is insufficient to determine mass using time-of-flight and direct-energy measurements. Highly-developed magnetic spectrometers, such as those discussed in the review ${ }^{13}$ by Enge, allow analysis of the heavier fragments by dispersing across the focal plane, depending on various properties, but they still have inadequate beam rejection at $0^{\circ}$. Devices such as beam filters or velocity selectors have been developed to separate the products of interest from the beam, but they leave the balance of the analysis to downstream detectors, which still lack energy resolution for heavy fragments. Only in recent years has there been an effort 10 combinc the beam-rejection function and the analysis function into a single "recoil spectrometer."

Some discussion of the most current efforts in the field are needed for comparison to the spectrometer discussed here. A velocity filter (SHIP) $)^{16}$ is included in the discussion. SHIP is included for its success in the limited class of experiments for which it was designed. Three full-recoil spectrometers (University of Rochester," Daresbury RMS, ${ }^{4}$ and the Laboratori Nazionali di Legnaro (LNL) RMS at Padova, Italy ${ }^{5}$ ] and a reaction separator [RPMS at NSCL $\mathrm{MSU}^{7}$ ] will be discussed, as they are working systems or are under construction. The FMA ${ }^{7}$ at ANL will be discussed in conjunction with the Legnaro device, as they are virtually identical. Several spectrometers will not be discussed, but are important to the current work in nuclear physics. IARA 17 is being built at Munich, MARS ${ }^{8}$ at Texas A\&M; also, the LISE ${ }^{18}$ and SPEG ${ }^{19}$ spectrometers are in use at GANIL.

\subsection{A Designs with Velocity Filters}

The present SHIP is a velocity filter ( $Q Q Q E D D D D E Q Q Q)$ at the Gesellschaft für Schwerionenforschung, Darmstadt, FRG. It is the most outstanding example of a heamseparation device for heavy-ion physics. The beam rejection ranges from $10^{12}-1()^{4}$ with ant acceptance of $2.7 \mathrm{msr}$ and a dispersion of $2.2 \mathrm{~mm} / \%$. As can immediately be seen, the final identification of the reaction products is almost totally dependent upon the detector at the focal plane. ${ }^{20}$ SHIP has rigidity $\left(E_{\rho}=20\right) \mathrm{MV}=10 \mathrm{MeV} / q, B_{\rho}=12 \mathrm{k}(\mathrm{i}-11 \mathrm{~m}=6.9 .5$ $\mathrm{MeV}$-nucleon $/ q^{2}$ ) in the range being discussed for the proposed spectrometer. The outstanding success of the work at GSI with SHilP is an excellent illustration of the matching of the spectrometer to the facility and the program for which it is planned. SHIP was built primarily to search for new elements, including super-heavy ones, and to explore particledecay modes of nuclei very far from stability. These could be identified by implanting the recoil products in a charged-particle detector and studying their particle-decay modes. Inverse reactions cannot be used at SHIP.

The RPMS at MSU6 is by far the most rigid of the systems to be discussed. The

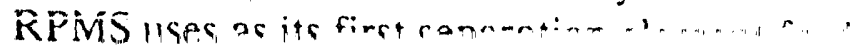


vantage that it can be "iuned" to pass a certain velocity. The condition on this velocity is $v=E / B$; thus, the electric field is not designed to have one value per product on produce an orbit through the machine. High-rigidity ions will pass with a low dispersion, and lowrigidity ions will pass with a high dispersion. This initially seems to be an excellent device, but some high prices are paid for these properties. Following the wein filter are nine quadrupoles and a dipole ( $Q Q Q W Q Q Q Q Q Q D Q Q Q)$. The chromatic aherration in this system is large, and, if completely cancelled, the higher-order terms become a problem. This spreads the line widths to the point that the mass resolution is 100 for $8 \%$ and $2(x)$ for $\pm 4 \%$ in $\Delta E$. This is for a recoil energy of a maximum of $30 \mathrm{MeV} /$ nucleon. The solid-angle acceptance is $1 \mathrm{msr}$. Although a good system for fragmentation or reaction studies, it is limited for other studies.

The RMS Daresbury ( $Q Q Q W W Q Q Q S S D Q Q Q$ ) is very similar to the MSU design. The velocity selector is separated into two parts, allowing the primary beam to be dumped without striking the electric plates. The solidangle acceptance is about 1 msr, and sextupoles have been added to correct some of the aberrations. The velocity acceptance is $\pm 2 \%$ with a mass dispersion of $10 \mathrm{~mm} / \%$. The use of an eiegani and complex detector at the focal plane enhances the recoil identification, so the low mass resolution is somewhat misleading. For spectroscopy work, the target is surrounded by an array of compton-suppressed Ge detectors. Future plans call for the use of a Ge ball arrangement of twenty detectors called Poly-TESSA. However, the $35-\mathrm{cm}$ distance from the target to the first element may present problems.

\subsection{B Zero-Energy-Dispersion Designs}

The discussion will now turn to the three other spectrometers and what can be gained from their design and operation. The design of the RMS at Rochester," the RMS at Legnaro, 5 and the FMA at $\triangle N^{6}{ }^{6}$ are all based upon the same premise. This premise is that by having a system with no spatial or angular energy dispersion, the energy aberrations will not be present or be very large for the system. Thus, by having an energy focus, both $\left(x / \delta_{E}\right)$ and $\left(\theta / \delta_{E}\right)$ vanish $\left(\delta_{E}\right.$ is the fractional energy dispersion $\left.\wedge E / E\right)$. The selection of an electric dipole before and after a magnetic dipole (EDE) is tice configuration used to produce an energy focus.

The differences between these spectrometers involve how the higher-order corrections are applied and how the intermediate optic constraints result in different focusing and beam rejection. All designs use a split-cylindrical electrostatic deflector. The magnetic dipole separates the two parts of the electric deflector. The high separation of the reaction products and the elastically-scattered beam particles is achieved because of this configuration. The momenta of the beam particles and the fusion reaction products (or $p / q$ ) are very close, while their $(E / q)$ are quite different. The electric deflector disperses based upon energy $(E / q)$, thus allowing beam and reaction-product separation to be a maximum. The immediate criticism of this design is that the primary heam strikes the first positive-potential deflection plate in the system. However, the Rochester RMS has been working in this manner for several years with no problems, so this is not considered to be an issue.

The Legnaro spectrometer has heen designed to minimize and correct for higherorder aberrations as much as possible. (This is not true of the Rochester spectrometer.) The Legnaro design (QQESDSE) uses only the two quadrupoles to focus through the entire spectrometer. No intermediate focus is formed. There is an energy focus, but this does not 
the beam extent and also keep the hicher-order aberrations as small as possible. In the: dispersive plane the focus is quite good, but in the vertical plan the beam size is approxi. mately $6 \mathrm{~cm}$. The second-order corrections that are made are used in part to make the mass-focal plane tilt go to zero. The Rochester design uses a quadrupole triplet for both entrance and exit to the spectrometer (QQQEDEQQQ). The final triplet both magnifies the mass focus and the dispersion preceding it and also brings about a spatial focus at the focal plane position.

The FMA at Argonne is virtually identical to the one at Legnaro in that it is a $Q Q E D E Q Q$ configuration. The sextupoles present in the Iegnaro design are repliced by al curved poleface boundary on the entral dipole. The final yuadrupole doublet is used 10 reduce the vertical beam size to approximately $1.5 \mathrm{~cm}$.

Several general comments on all three of these spectrometers can be grouped together. First, the high-beam rejection is based on multiple scattering within the spectrometer if the heam strikes the first positive plate of the split electric dipole. For so-called normal reactions (projectile particle lighter than target nuclei), this will always work. If the beam does not strike the first electric dipole plates, this rejection is lost. If the energy of the beam particles $\left(E_{b}\right)$ and the energy of the reaction recoils $\left(E_{r}\right)$ both lie within the energy acceptance of the spectrometer, both types of particles will be focused on the focal plane if the rigidity of the spectrometer is sufficiemly high to handle the recoils. Obviously, the energy of the recoils can never exced the energy of the beam particles, so this case need not be considered. Between the two extremes of $E_{b}>>E_{\text {r }}$ and $E_{b} \approx E_{r}$ a very large variety of reactions, focusing conditions, and heam rejection levels can be devised. The net result is that not all or necessarily even a large number of reactions, particularly inverse reactions, can be used in a spectrometer of this type.

Second, the rigidity of these spectrometers is relatively low at $6 \mathrm{MeV} / Q$ for the Rochester RMS and $9 \mathrm{MeV} / Q$ for Legnaro and Argonne machines. This puts an upper limit on the reactions that can be studied with these spectrometers. Any reaction producing a recoil of $\approx 5 \mathrm{MeV} /$ nucleon cannot be studied in these systems (even assuming $Z=N$ and complete stripping). Thus, rigidity is importint if the spectrometer is to cover a broad range of reactions.

The discussion has been centered on comparing the design of velocity filters or other recoil mass spectrometers to the design presented here. Some comments comparing a RMS with more traditional magnetic spectrometers (BRS, Q3D) or isofope separators need to be made. The rigidity and dispersion desired can be obtained in these spectrometers, but the beam rejection at $0^{\circ}$ and large solid angle are problems that cannot be overcome together. Although mass separators can be made to have large acceptance, the problems of different ionization efficiency for different reaction products, the loss of correlation between information at the arget location and the separator focal plane, and the long hold-up time of the species in the ion-source are not present (and therefore not problems) in the RMS. Considering these spectrometers, separators, and the other RMS designs discussed, the design effort here is for a spectrometer based upon the Rochester approach, but with significant improvements. Effort was made to remove the second-order aberrations but without the loss in spatial focusing. Rigidity will be high to match the HHIRF accelerators as well as possible. 


\subsection{DESIGN INIROIDUCTION}

Careful attention was given to match the RMS to all the beams available from HHIRF accelerators, including those with the highest energy, and also massive particles for use in inverse reactions. For the tandem, the highest energy for fully stripped nuclei would be about $25 \mathrm{MeV} / q$ for $N=Z$. For the electrostatic deflectors used in this design, the maximum energy is about $15 \mathrm{MeV} / q$ or $7.5 \mathrm{MeV} /$ nucleon for $N=Z$ and fully-stripped recoils. This shows the need for the high rigidity. This last consideration is in contrast to some present and proposed RMS facilities.

In designing any spectrometer, close attention must be given to the broad view of what experiments are to be performed with the system. Initially, the primary expectation for the RMS was to study exotic nuclei from normal reactions with a heam that was lighter than the target nuclei. Some attention was given to inverse reactions but more in the nature of those that would have the beam focused onto the focal plane. Since that original design effcrt, inverse reactions have heen reconsidered. The current view is that "all" inverse reactions must be handled by the RMS, and the case of the primary heam reaching the focal plane is not acceptable. This is to say that the high beam rejection must work equally well for inverse reactions. The kinematic advantage in using inverse reactions is simply too large to lose for any reaction.

A completely new consideration for the spectrometer was the GAMMASPIIERE: project. 21 At the time the idea for GAMMASIIHERE was formulated, none of the recoil spectrometers was considered to be important to its mission. After some consideration and the appearance of early results from experiments at Rochester and Dareshury, the importance of the RMS to GAMMASPIIERE was seen. The problem was that none of the existing or proposed spectrometers could operate at the large image or object distances required by GAMMASPHERE without significant loss of performance.

With these new conditions in mind, a modification to the RMS was sought that would allow use of all inverse reactions, have large image and object distances, and meet the high rigidity needs of HHIRF. The result that is presented here is the spectrometer that is currently being built for use at HHIRF.

The general description of the modification is that a momentum achromat has becu added to the front of the original RMS design. This is shown in the element layout in Fig. 1.

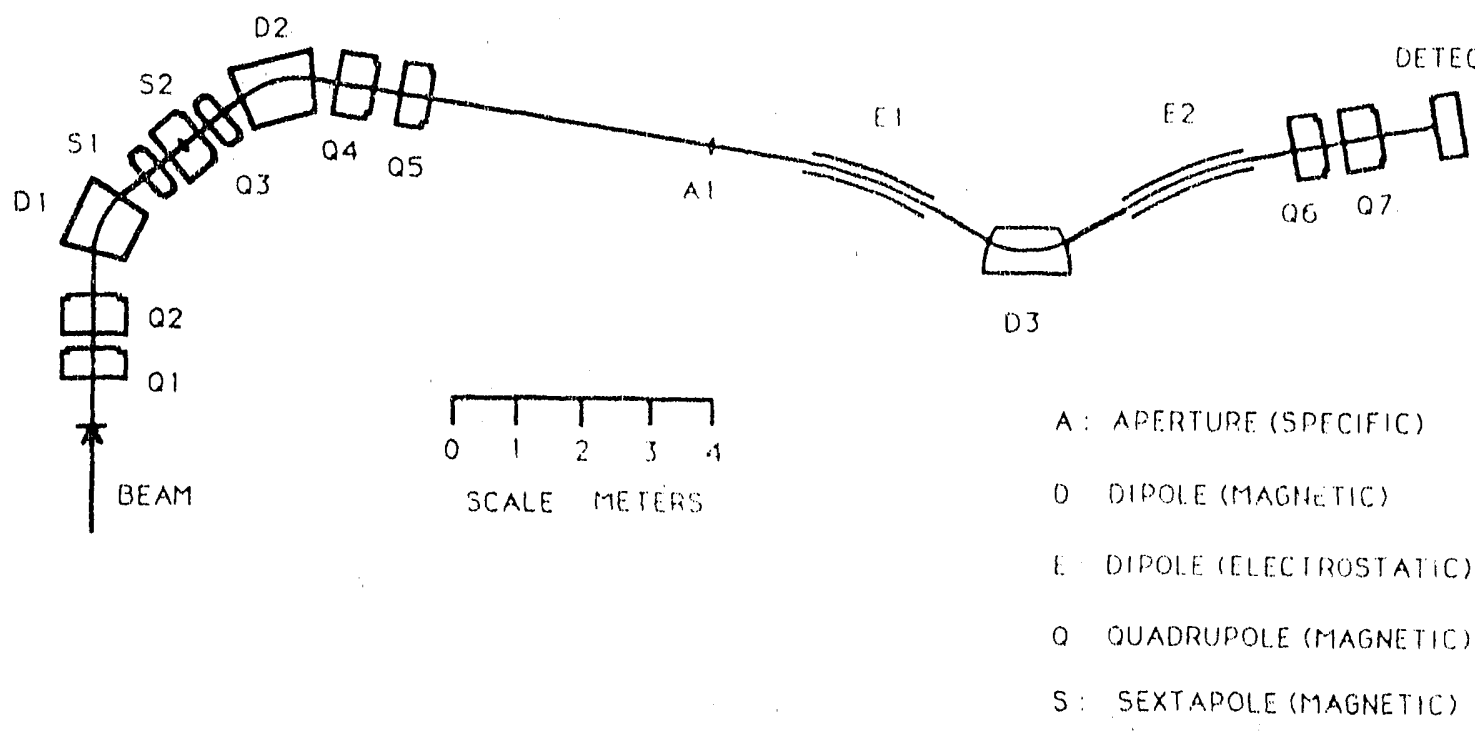




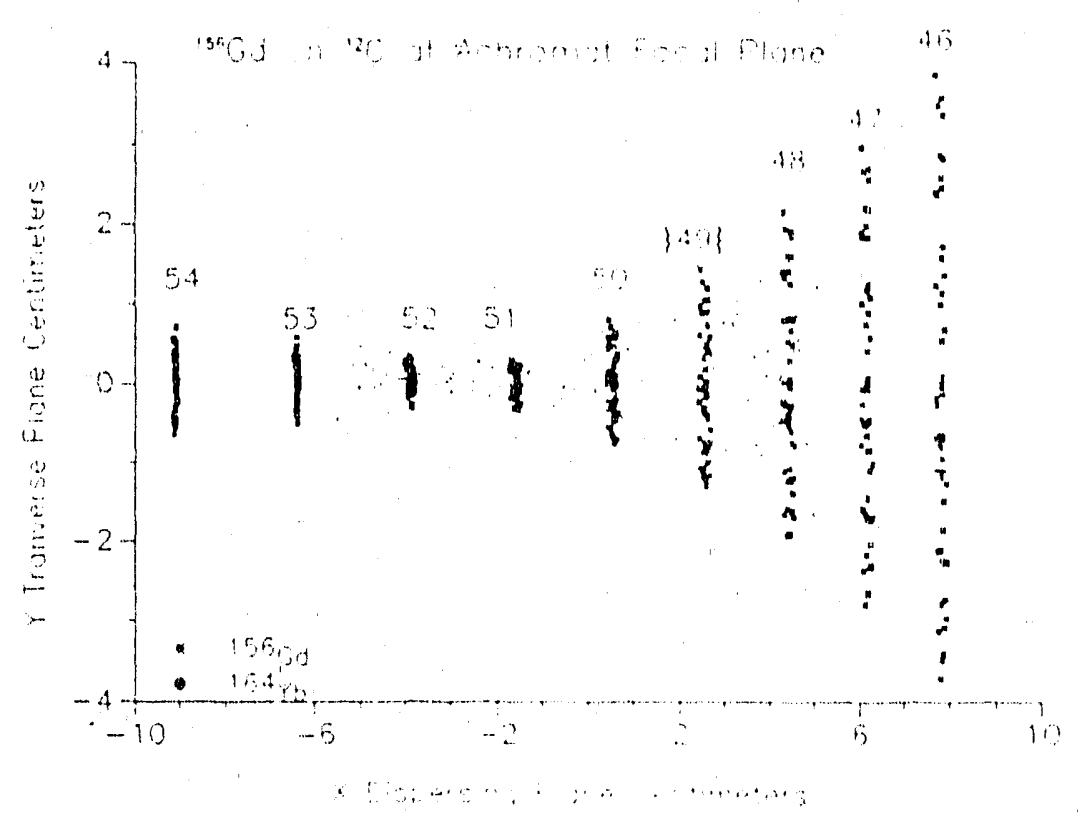

Figure 3. The reaction products and bean particles for the reaction, 154(id(12C,4n) lexty, at the focal plane the achromat. The most probable charge state for the beam is $\{49\}$. A pereent of the 16 yb would be stopped with the beam, but this is a negligible amount.

sults from the focal plane being at a $70^{\circ}$ angle to the beam axis, whereas the calculation is for a plane perpendicular to the beam axis. The heam particles can easily he stopped, while the reaction products are passed with only at small loss.

\subsection{A Target Location and First Quadrupoles}

The general view of an experiment with an RMS is that the RMS is but one element in the experiment. Detectors (Ge, Si, plastic, NaI, liquid scintillators, gas proportional counters; in singles or various array configurations will be used with the spectrometer. $\wedge$ variety of different detectors may be used at the focal plane for different purposes. How. ever, detectors around the target itself are essential to most experiments, so the initial consideration is the target location. The proposed use of GAMMASPIIERE with the RMS puts severe requirements on both the image and object distances. The physical distance from the target position to the first spectrometer element (in this case a quadrupole) needs to be as large as possible. The other comsiderations affecting the target location are llo solid angle subtended by the spectrometer and the magnification of the instrument. For energetic reactions above the coulomb harrier, the strong forward focusing of the emilted particles requires that particle detectors be placed between the target and the spectrometer. In particular, this can be a problem for neutron detectors, as the size of the detector is determined by the interaction distance of the neutron. The target distance chosen in the present case is $75 \mathrm{~cm}$. This gives good space for both $y$-ray and neutron detectors. Moreover, this distance with quadrupoles that have an aperture with a diameter of $20 \mathrm{~cm}$ still gives a large solid angle of $15 \mathrm{msr}$. The angular acceptance is not symmetrical, as the dispersive plane has an angular acceptance that is one-third that of the vertical plane. Any slits used to define the solid angle of the spectrometer will be positioned at the entrance (o) the first quadrupole. The first lens is diverging in the dispersive plane, and the second lens

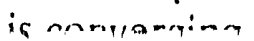


The optics of the achromat are parallel-to-parallel in the dispersive plane. At the focal plane of the achromat, the center of qualrupole \#3 (Fig. 1), the dispersion is a $10 \mathrm{~mm} / \%$ and the resolving power is $\approx 350$ at the full solid angle, $n=25 \mathrm{msr}$. The achromat is composed of three quadrupoles and two dipoles. The dipoles are $50^{\circ}$ in hend angle with hoth dispersive in the same direction. The two entrance quadrupoles have 20-cm apertures, and the third quadrupole has an aperture of $30 \mathrm{~cm}$. This achromat precedes a Rochester-style RMS (i.e., a split electric dipole) that is basically the original design.

The achromat is designed such that there is a focus formed between the quadrupoles and the first electrostatic deflector. At this point the beam is completely removed and an image of the target spot is reformed. Since the distance between the quadrupole and the electrostatic deflector is $6 \mathrm{~m}$, with this focus occurring $4.5 \mathrm{~m}$ after the quadrupole, the achromat can be used independently of the rest of the RMS. This provides a momentum achromat of rigidity of $25 \mathrm{MeV} /$ mucleon and a solid angle acceptance of 25 msr.

To understand the advantage and power of the addition of the achromat, consider the following example of an inverse reaction at $5 \mathrm{MeV} /$ nucleon: $156 \mathrm{Gd}$ on $12 \mathrm{C}$. In this case, $164 \mathrm{Yb}$ is the reaction product of interest. For a RMS of the Rochester style, both the primary beam and the reaction products pass through the first electrostatic deflector unscattered. In the case of the ${ }^{156} \mathrm{Gd}$ on ${ }^{12} \mathrm{C}$, the most probable charge states of the heam actually result in $M / Q$ ratios that are focused at the focal plane. This is shown in Fig. 2 as calculated for the original design without the achromat. Charge states 47 and 48 reach the focal plane of the RMS if they are not stopped in the achromat.

Figure 3 shows the beam particles and the reaction products for the ${ }^{156} \mathrm{Cd}$ on ${ }^{12} \mathrm{C}$ case at the focal plane of the achromat. The most probable charge states are shown but without being weighted. That is to say, an equal number of rays are used in each charge state calculation, and the figure does not represent the most probable population of each charge state. The reaction products clearly fill the space available at the focal plane, while the beam particles are focused into lines. The change in height in the $y$ plane for each mass re-

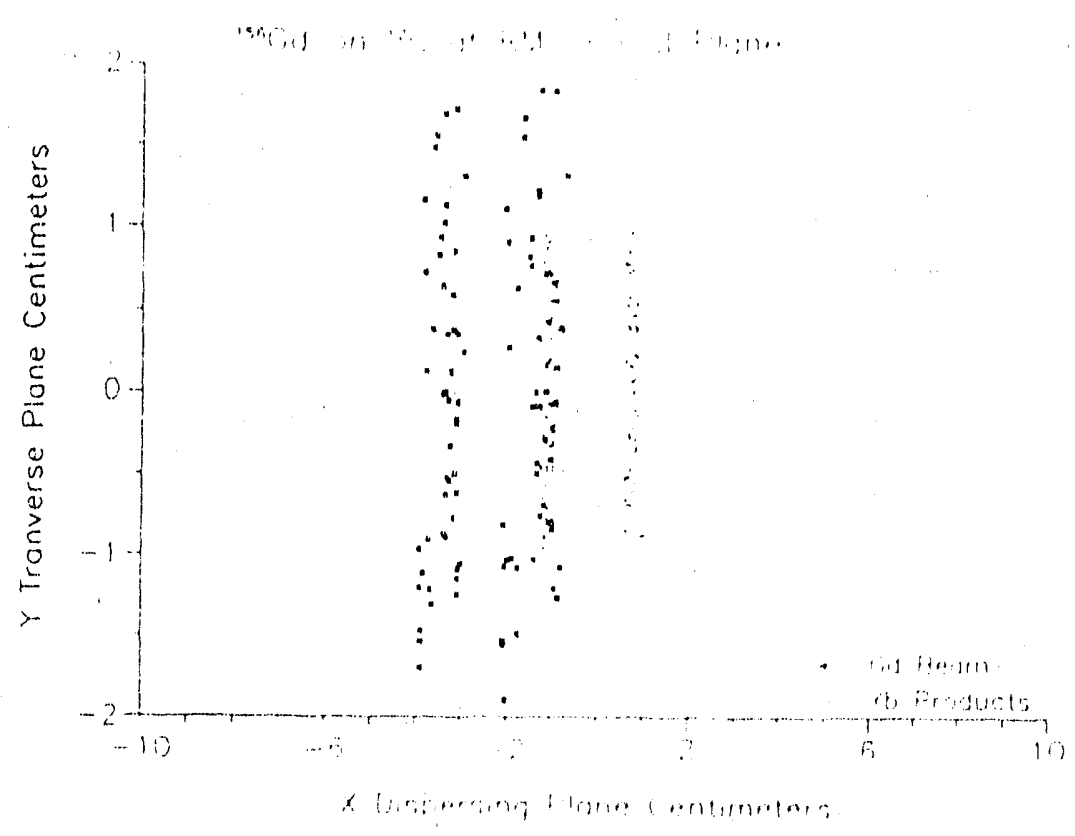

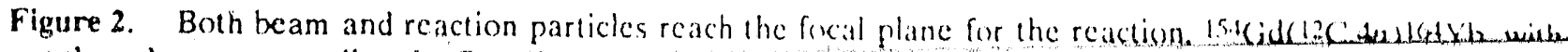




\subsection{B Electric-Magnetic-Electric Dipoles}

The choice made for the present design is an electric-magnetic-electric dipole configuration following the achromat. The physical dimensions of these elements are $20^{\circ}$ electrostatic deflectors with a radius of $6(0) \mathrm{cm}$ and a separation of $10 \mathrm{~cm}$. The deflectors are separated by a magnetic dipole with a $50.0^{\circ}$ bend angle and a radius of $140 \mathrm{~cm}$. The gap of the magnet is $10 \mathrm{~cm}$, with the pole faces having no curvature. The magnet is weakly focusing with shim angles, $a=b=15^{\circ}$. The electric deflectors are planned to have initially a maximum field of $40 \mathrm{kV} / \mathrm{cm}$, which would yicld an electric rigidity, $E_{\rho}=24 \mathrm{MV}=12$ $\mathrm{MeV} / q$. The final goal is to condition the plates 10 hold $50 \mathrm{kV} / \mathrm{cm}$. This would give $E_{n}=$ $30 \mathrm{MV}=15 \mathrm{MeV} / q$.

\subsection{Final Quadrupole Doublet}

At this point, the system is a mass spectrometer. At the exit of the second electric deflector, a triple focus occurs in the dispersive plane. By adding the quadrupole doublet, a vertical focus can be obtained, the mass dispersion increased by changing the magnification. These improvements have their price in other aberrations becoming larger, but some of the worst of these can be corrected in the focal-plane detector.

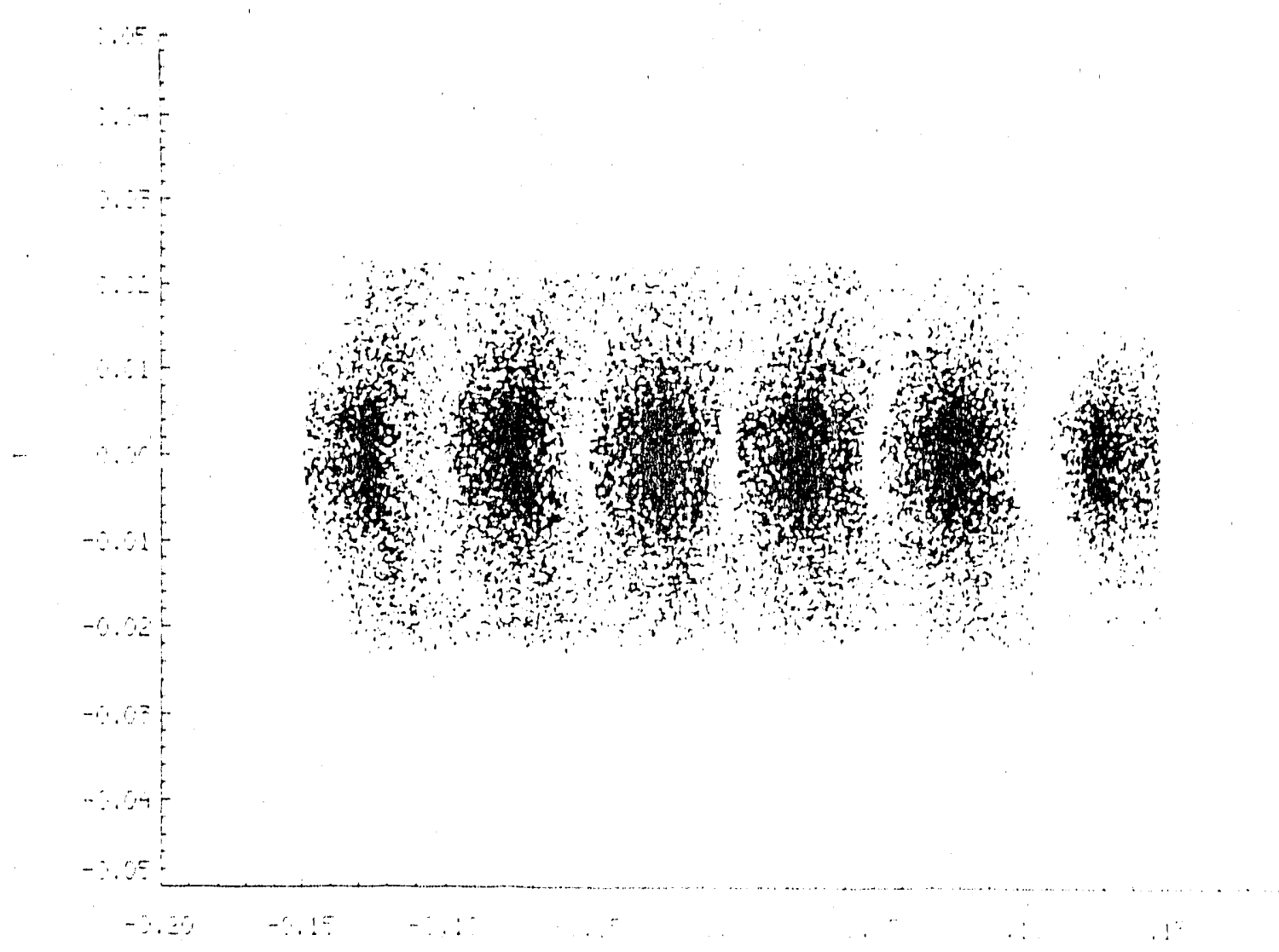

Figure 4. The position in $X, Y$ at the focal plance of the spectenncter for different m/l values aliout the cen-

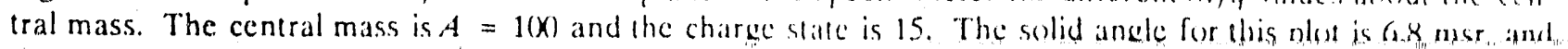


In summary, the RMS will have the following characteristics:

a) a large-acceptance solid angle of up to $15 \mathrm{msr}$

b) an energy range $(\Delta E)$ of $3.5 \%$

c) a mass-to-charge ratio range $(\mathrm{m} / \mathrm{q})$ of $\pm 5 \%$

d) a good mass resolution ( $m / \Delta m \neq 770)$ (FWHM) at 10.0 msr and +540 (FWHM) at $15.0 \mathrm{msr}$ (Fig. 4 shows the focal plane resolution for $10.0 \mathrm{mss}$.)

e) a mass dispersion $\left(x / \delta_{m}\right)$ of $\times 43 \mathrm{~mm} / \%\left(\delta_{m}=\Delta m / m\right.$ or the fractional mass deviation in percent)

f) a magnification at the focal plane for the reaction products

g) an excellent primary beam rejection $\left(-10^{13} \mathrm{in}\right.$ most cases) at ()$^{\circ}$

h) a large target-to-first-quadrupole distance of $75 \mathrm{~cm}$

The spectrometer is to be comprised of seven quadrupoles, two electrostatic deflectors, and three magnetic dipoles in a $Q Q D S Q S D Q Q E D E Q Q$ configuration. The electrical rigidity $\left(E_{\rho}\right)$ is initially to be $12 \mathrm{MeV} / q$, with the design goal being $15 \mathrm{MeV} / q$. The magnetic rigidity $\left(B_{\rho}\right)$ for the dipoles is $20 \mathrm{kC}-\mathrm{m}(2 \mathrm{Tm})$ maximum with corresponding rigitity planned for the quadrupoles to match closely the spectrometer to the accelerators at ORNL.

The total transmission of the spectrometer is also very good, with approximately $90 \%$ of the reaction products reaching the focal plane at the 10.()-msr solid angle. Continuing efforts to improve the spectrometer will occur during the engineering-design phase and with the establistment of the specifications. Final adjustments and refinements will occur to the spectrometer as fabrication, assembly, and testing occur.

\subsection{CONCLUSION}

The fiald of heavy-ion physics is developing its own instruments to address the problems and experiments unique to this area. A wide range of heavy-ion-bhysics experiments can be done with an RMS, as suggested in Table 1 . A list of some experiments proposed for the HHIRF RMS is given in Table l. Some of these experiments can be carried out on other types of devices, but only an RMS can perform the many different experiments. In particular, it is essential for many of the proposed experiments on short-lived and/or low-cross-section products. To document this, since it became operational at Rochester, the RMS has been used in 50 -70)\% of all experimental ruming time of the accelerator. The large solid angle, high heam rejection, large mass dispersion, lange energy acceptance, and large mass resolution are combined into a device that has uses in nuclearstructure studies (be it tinrough traditional spectroscopy of radioactive decay, in-heam high spin, or the new decay modes of exotic nuclei), reaction studies, fusion studics, and radiuatctive-beam production. The RMS proposed here has the capabilities to perform these functions. The design is new in the sense that a configuration was selected and calculations were performed to match the spectrometer to the accelerators at MHIRF so that the experimental program outlined could be performed. The rigidity chosen is within the limits of what can be produced today, but the caphability of incorporating improvements is present in the design. 
Table I. Research with an RMS

Radioactive decay of proton- and neutron-rich exotic nuclei.

Generally inaccessible nuclei

Weak reaction channels $(* 1 \mathrm{mb})$

Short half-lives $(\approx 1(x) \mathrm{ms})$

Difficultly-ionized species (e.g., Zr, La, W, Ta)

Exotic decay modes - at (or past) proton/neutron drip lines

$\beta$-delayed particle emission

Super-heavy nuclei

Low-lying, excited-, and ground-state propertics

High-Spin studies of neutron-and proton-rich nuclei

Nuclei with weak reaction channels

Continuum $\gamma$-ray studies

Band structure of weakly-populated states

Alignment at high spins

Average internal conversion

Fusion studies

Measure fusion cross-sections

Fusion resonance

Reaction studies

Quasi-elastic

Fragmentation

Massive transfer reactions

Resonances

Radioactive beams

Coulomb re-excitation of reaction products

NMR studies

Implantation at lattice sites

Characterize transport techniques

Atomic spectroscopy of highly-ionized atoms 


\section{ACKNOWLEDGMEN'TS}

The funding for our RMS is heing provided hy the U.S. Department of Inergy, a major contribution from the State of Tennessee, significatt contributions from l(bahe itidtional Engineering Laboratory, Vanderbilt University, the University of lennessecKnoxville, Oak Ridge Associated Universities, and the Director's Office of Oak Ridge National Laboratory; also, contriburions from UNISOR and its inembers, Georgia Institute of Technology, Mississippi State University, and the University of Maryland. This work wils supported in part by the U. S. Department of Energy under contracts No. DE-A(C)7. 76ID01570, DE-FG05-86ER40256, and DE-F(0)-88ER4(2407.

\section{REFERENCES}

1. J. H. Hamilion et al, Rep. Prog. Phys. $48,6.31$ (198.5).

2. J. H. Hamilton et al, High Angular Momentum Properries of Nuctei, Ed. hy N. R. Johnson (Harwernl. New York, 1982), n. 227.

3. J. H. Hamilton, Treatise on Heary Ion Science, 8, Ed. by A. Bromley (Plenum, New York, 108,4), 1. 2.

4. H. G. Price, Daresbury Lab Repore, DL./NUCL/RIY (1979)).

5. P. Spolaore et al., Nucl. Instr. Meth. A238, 381 (1985); P. Spolaore et al., Nuovo (iim. 81A, 351 (19).4).

6. L. H. Harwood and J.A. Nolen, Jr., Nucl. Insir. Mcth. 186, 435 (1)R1).

7. C. N. Davids and J.D. Larson, Nucl. Instr. Meth. BHh-41, 1224 (198')).

8. T. M. Cormicr, private communication.

9. T. M. Cormier and P.M. Stwertka, Nucl. Instr. Meth. 184, 423 (11)81); T. M. Cormicr et al., Nucl. Instr. Meth. 212, 185 (1983).

10. R. B. Piercy et al., "Conference on Instrumentation for Heary-lon Nuclear Rescarch," ORNi, Repo:1 CONF-841005, 27 (1984).

11. J. H. Hamilton, Fundamental Problems in Heayyion Collisions, Ed. hy N. Cindro, W. (ircincr, and R. Caplar (Workd Scientific Publishing, Singapore, 1884), p. 111

12. S. Wen et al., J. Phys. G 11 L173 (1985); S. J. Rohinson el all, Bull. Am. Phys. Sire. 31), 726 (11485).

13. H. A. Engc, Nucl. Instr. Meth. 186, 413 (1981).

14. H. A. Enge, Nucl. Mnstr. Meth. 162, 161 (1070)

15. K. Sistemich, Nucl. Instr. Meth. 139, 2(1).3(1976).

16. G. Münzenberg et al., Nucl. Instr. Meth. 161, 65 (197\%).

17. W. W'ilhelm, privale communication.

18. D. Guillemaud-Mueller et al., Proceedings of the Sh Intemational Conference on Nuclit Fur From .Su-

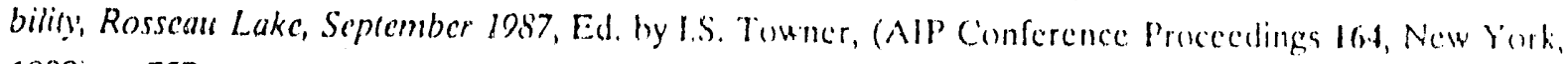
1988), p. 757.

19. Zahn Wemlong et al., Nowelle de (SANIL 25, 22 (11)48).

20. G. Münzenberg et al., Nucl. Instr. Meth. 186,423 (1981).

21. I.-Y. Lee, Chapter 15 in This Book. 

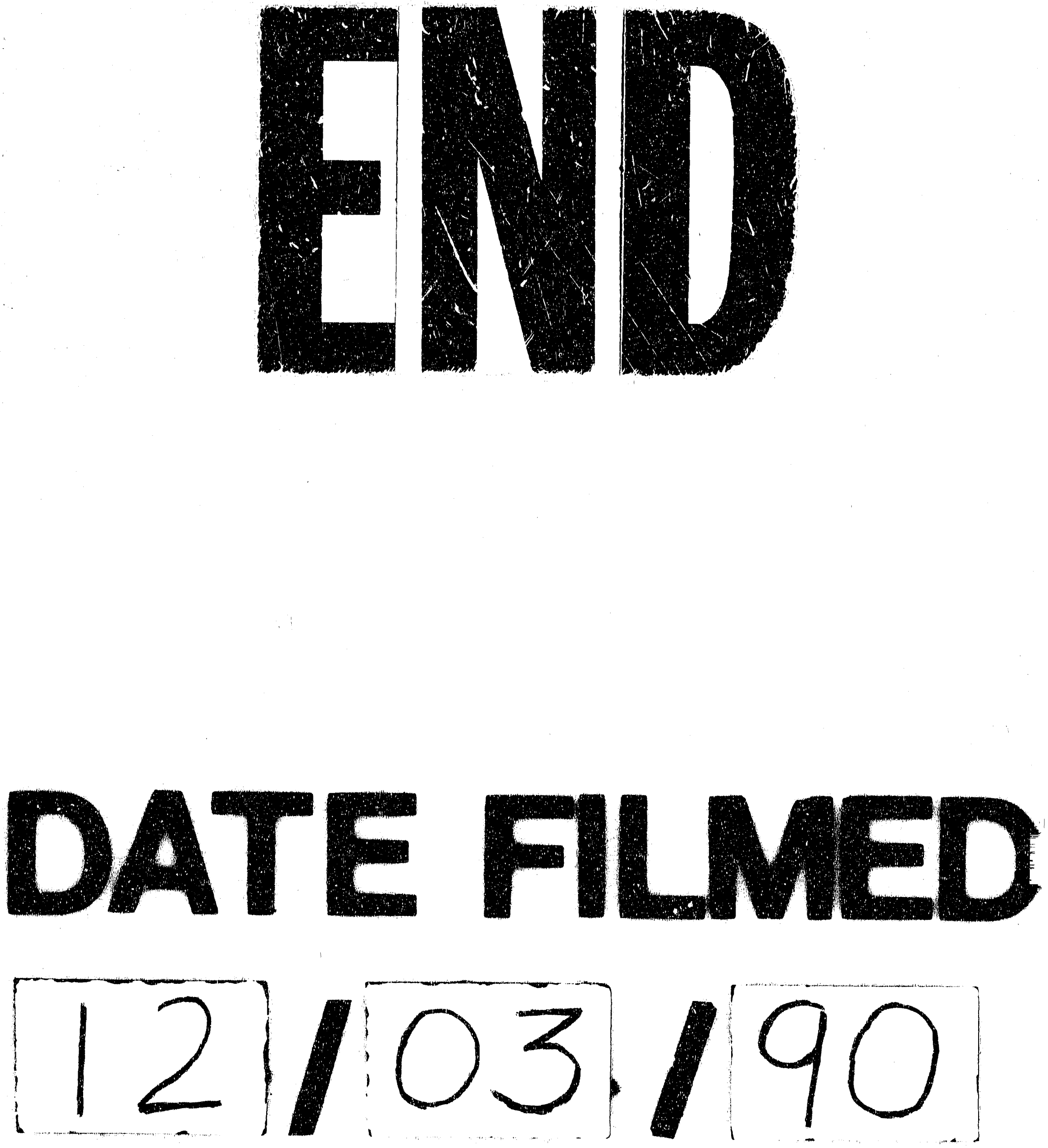
